\title{
Framework for Security Analysis and Access Control in a Distributed Service Medical Imaging Network
}

\author{
IAN R GREENSHIELDS, ZHIHONG YANG \\ Department of Computer Science and Engineering, the University of Connecticut, U-1031, \\ UConn, Storrs, CT 06269-1031, USA \{ ian, zhyang\}@engr.uconn.edu
}

Key words: Hierarchical Access Control, DSMIN, Active Streams, Agent Ferry

Abstract: A data security system framework on a Distributed Service Medical Imaging Network (DSMIN) is given in this paper. The DSMIN was previously proposed based on the notion that a general distributed services medical network is the future of a computational medicine model in which imaging plays a significant role. In the DSMIN, both data and code migrate through the network with a goal of optimizing performance while maintaining security. After an empirical analysis of the security demands of this system, a hierarchical access control model that combines user authentication and namespace interposition is proposed to deal with security in the DSMIN. Agent technology is adapted to fulfill the security demands in two ways. First, an active stream enabled by agents combines image compression and encryption when the image is transmitted over the network. Active streams are built on top of the transportation layer. Secondly, data and code are shipped by an agent ferry, which wraps the raw data on the channel. With in-built mechanisms in the agent ferry, access control logic is implemented, and name space interposition is enforced in combination with existing security technologies.

The original version of this chapter was revised: The copyright line was incorrect. This has been corrected. The Erratum to this chapter is available at DOI: 10.1007/978-0-387-35515-3_53 


\section{DSMIN AND SECURITY}

There is a significant growth in the deployment of IT in the health care industry, although it is generally perceived that the health care industry's use of information technology is 10-15 year behind sectors such as banking, airlines and the auto industry [1]. The integration of these two disciplines will not only revolutionizes health care delivery, opening up the industry to a broad area of information technology. Given the above background, the Distributed Service Medical Imaging Network (DSMIN) [2][3] is a new computational model specifically targeted to health delivery systems in which a significant pportion of the data carried is medical imagery. The DSMIN employs a suite of modern information technologies, notably distributed technologies, to medical imaging processing. It is the provision of a distributed, scaleable medical imaging system deployed over cells of clustered heterogeneous workstations connected by narrow band and broadband networks. It has two roles: distributing imaging services (in the sense of new imaging code) to a user base including physicians, and transporting images (and associated ancillary data) between clients (physicians in local offices, hospital-based physicians, insurance provider etc.) in the system.

In this paper we concentrate on some of the data security aspects of the DSMIN. Environments such as a DSMIN bring challenges to healthcare management, technical developers and the user community (including both physicians and patients). In this section, the computation model of the DSMIN will be discussed because this is the base that the security solutions are built on top of. The security demands of the DSMIN arise from its broad user community, which includes patients, physicians, technicians and developers, and these demands are significantly different from most conventional systems owing to the massive disparities in the roles of the users in the system. Section two is dedicated to the security demand and analysis in DSMIN. We propose a hierarchical access control to enforce these security requirements in section three. Section four addresses the security technology currently available in networks. This is the basic technology we deploy in the DSMIN. Section five proposes agent ferry architecture that is the facility that will used to implement access control and user authentication in the DSMIN. Another technology called active streams that builds a secure transport layer on top of the network is discussed in section five. We discuss open issues and our preliminary observations in section six. 


\subsection{Background}

Two broad categories of research dominate the literature in computer based medical systems. The first is that of medical information management and delivery systems (e.g. hospital management systems, online medical information access systems, patient record and medical data exchange systems and collaborative diagnosis systems). Networking and database technologies are broadly applied in this first category. The second is medical image data processing and analysis. The technologies that dominate here include classification, pattern recognition, volume rendering, virtual reality and image processing which are used to extract diagnostic information from various data generated in medical practice. These data include Magnetic Resonance Imaging (MRI), electrocardiographs (ECG), digital and plain-film mammography, computed tomography (CT) and digital and plain-film radiographs. It has always been difficult to get research algorithms in imaging out from the laboratory into the field. Here, just as in every other software application domain, there is an ongoing (and difficult) issue of code-reuse that needs addressing. Another problem is that these medical data analysis and processing algorithms often require massive amounts of computational resources, including compute cycles, memory and network (and I/O) bandwidth.

There is an enormous literature associated with these two computational medicine domains. Examples include telemedicine, teleradiology, health care delivery systems for patient education and treatment, patient record exchange system, and the application of virtual reality and telerobotic technologies for teaching and learning surgical procedures both locally and remotely [4][5][6][7][8]. The DSMIN might be most closely associated with teleradiology, although it tends to superset most current teleradiological systems. If we consider so-called "value-added" teleradiological systems (i.e, those systems which support an analytic component as well as a distribution component), the common computational model is classical client-server, in which the client side transmits data for remote analysis by the server. The DSMIN generalizes the server side of this kind of teleradiology.

We proposed the DSMIN as an infrastructure for addressing some of these problems. . Specifically, we are interested in satisfying some of the following requirements: -

1. Code re-use (distribution of algorithms) in a natural way

2. Platform independence

3. Seamless scheduling of code on the total compute fabric. which constitutes the DSMIN 
4. Dynamic monitoring of this fabric (dynamic addition and deletion of resources)

5. Seamless, secure data transit on the DSMIN

6. Hierarchical access authority

7. Dynamic negotiation of channel properties relative to data distribution, especially for mobile components of the DSMIN

The algorithms in DSMIN are implemented as distributed components, thus reuse is implicit in their design, and they automatically become part of a super fat (distributed) server which services medical image processing. Naturally, intercomponent interactions are normal. Given this, data formats have to be standardized so that data can traverse the system seamlessly. This is principally a syntactic issue, addressed by the DICOM imaging standards. Generally, services and data are not in the same locale when a service is invoked, and indeed it is not clear (at the time of invocation) whether it is the code which will migrate to the client or the client's data which will migrate. It is a specific design goal of the DSMIN that code (and data) migrates in an optimal way so those services are provided by the most appropriate part of the DSMIN fabric. We address this below.

\subsection{Computational model of DSMIN}

Figure 1 shows the network architecture of the DSMIN.

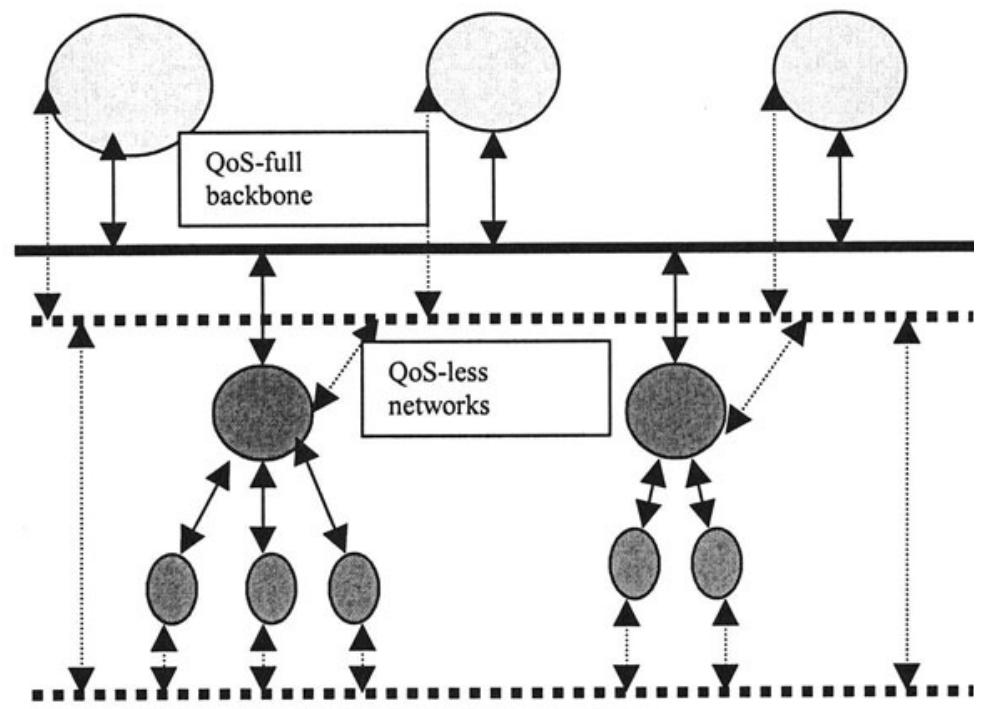

Figure 1 Network Architecture of DSMIN

Clearly, the DSMIN comprises a suite of local clusters interconnected via both QoS-less networks and a Qos-full backbone. 
In the DSMIN, the assumption is mad that the data and the technology that is able to process the data may be geographically separated. DSMIN clients make requests for data (which may be local or may be remote) and either makes explicit requests for services associated with this data, or have services attached to the data implicitly by the data owner. Both data and code are in motion in the DSMIN. We distinguish four broad categories of computation that can be invoked dependent on the client, the service requested, the dataset size and security attached to the data. These are: -

Traditional client-server. In this model, a remote service is both invoked remotely and satisfied remotely. Either the data is remote (at the server), or the data is tansferred to the server.

Traditional mobile code. Here, a remote service is dispatched to the client (as a piece of mobile code) and executed locally at the client. Typically, services in this model are computationally simple, and are associated with small datasets.

Local-cells. Parts of the fabric are designated as local cells (e.g., a cluster of workstations in a laboratory). More demanding tasks are distributed over a local cell using an agent model.

Broad collaboration. Here, a service is distributed as widely as possible to parts of the fabric, which are geographically widely distributed. Such services are usually processes in which CPU times are measured in minutes (or hours).

The service distribution architecture is innately tied to the data security architecture. This is our focus for the remainder of the paper.

\section{SECURITY ANALYSIS}

Security analysis addresses the potential risks faced by the system as well as the possible attacks that the system should be able to defend against. Compared to traditional healthcare delivery information systems, the security demands in the DSMIN are more complex, and involve more elements. In traditional healthcare delivery information systems, the security concern is mainly focussed on the privacy and confidentiality of patient data. Role-based security systems [9] are usually used to analyse information access and control in health care delivery information systems. The main point of this analysis is based on the observation that professional responsibility plays an important role in information access and control. The access to information is not simply based on the trustworthiness of the user but more on the user's need for information in order to perform the user's professional functions and duties. In this way, each individual's data access rights depend upon the relationship this person has with other medical 
personnel, as well as his/her assigned duties at different locations and times. The specifications of data access right are often content-dependent as well as time-dependent.

In the DSMIN, however, not only do patient records, but also the data processing technology (e.g. the source code and the executable code, and the computation resources themselves) need to be protected $[10][11][12]$. There are several reasons for the protection of the executable data. First, executable data represents are intellectual property, which may be subject to protection requirements. Secondly, these executables generate results that will be relied upon in making diagnoses, so the service's integrity through network transmission should be fully assured. Finally, mobile code must be protected from attack by malicious hosts.

\subsection{Hierarchical Access control}

Access control models the accessibility of system resources to different actors involved in the system. We propose a hierarchical access control model in the DSMIN [14]. In general, there are four principles in the DSMIN:

1. Clinically-qualified staff (e.g., physicians, nurses)

2.Non-clinically qualified medical staff (e.g., certain technicians, secretaries)

3. Non-medical staff (e.g., programmers)

4. Patients

Security is one of the most complex parts of the architecture. As indicated above, a host may wish to restrict the service locales that data (or even code) can be transmitted to, either to protect sensitive data, or to protect sensitive code. However, even when data can be transmitted, it most likely will require to be transmitted in encrypted form.

Encryption in the system comes in two methods: strong (hierarchical) encryption, and weak (image) encryption. Strong encryption is essentially conventional encryption, employing both shared and public/private key management together with authority monitoring. This encryption flavour is usually employed over textual data (Patient ID, patient records etc.), and is constructed in such a way to as to permit a document to be hierarchically encrypted. By this we mean the partitioning of a document into $Q$ ranked equivalence classes with the property that an authority with permission $P_{m}$ can decrypt all parts of the document encrypted at equivalence classes $E_{j}$ provided $\mathrm{m}>\mathrm{j}$. In this way, master key holders can decrypt the entire document, while more restricted users can only decrypt those parts of the document for which they hold the appropriate key authority. 
Image data usually does not require this kind of security. Among other things, image data is rarely identifiable with patients (PID data typically being kept separate), and the cost of encrypting a large image dataset can be significant. However, there may be reasons to convey image data in such a way as to make it difficult to view the data (albeit not cryptographically difficult). One way to do this is to exploit a compression technology (which would be used anyway) to simultaneously encrypt the image data. Wavelet compression of images is rapidly becoming a standard for image transport. If a codec is given sufficient latitude in choosing the mother wavelets (at each stage of the decomposition), a simple encryption scheme can be developed whereby the compressor chooses a sequence of base wavelets to effect the decomposition (perhaps even trying to adhere to a best-basis decomposition). This sequence is enveloped to the receiver so that the decompressing codec can reconstruct the data.

Throughout the system, data, as an independent entity, does not exist. No "raw" data can be communicated between any source and destination. Rather, an agent ferry that wraps the data must carry all data in motion in the system. Clients see only (and interact only) with these ferries. Figure 2 at the end of this paper illustrates the transfer protocol.

In a DSMIN setting, the system resources that need to be protected can be partitioned into two categories. The first one is application-independent. The concern of application independent object partitioning is to protect computer and its computation capability and file system and other functional parts. The granularity of partitioning is not fine enough to satisfy the needs of specific application. The application-specific partitioning is developed to distinct the security property of structured data, like patient record, to facility access control. DSMIN must combine those two categories in its security framework. The technologies that deal with application-independent access control are mainly the cryptography and a set of security services.

\section{AGENT FERRIES AND ACTIVE CHANNELS}

Authentication is dual here; all mobile code transported to clients is certified, but clients must also certify themselves (in their user role) to the ferries. Ferries use this user-role certification to perform authenticationbased name-space interposition in the methods they convey. All authentication slots are transaction limited (to the dataset ferried), and authentication ensures the user access to the data commensurate with his/her user role (position) in the hierarchy. Access control is implemented, as implied, by name-space interposition. Figure 3 at the end of this paper shows this interposition. 
Active channels are end-to-end channels set up for the active transport of imagery, and imaging code, overlaid with base security architecture. Active channels are self-monitoring, so that each channel

1. Maintains a dynamic model of channel activity (channels are not passive conduits of data).

2. May dynamically (re) negotiate channel properties (e.g., codecs).

3. Dynamically monitors channel QoS.

Essentially, an active channel is a peer-to-peer link that is agent-enabled at the source and the sink. In the simplest form, an active channel will negotiate codec charactersitics between the source and the sink, and (if necessary) the source will update the sink with the required codec the source believes to be most compatible with its goals for the channel. The obvious example is a wavelet-based codec, with decomposition levels and mother wavelets negotiated by the channel peers. Equivalently, the channel can negotiate to carry color if the channel's capacity permits, or to drop color (or alter image sizes) if the channel's characteristics change.

\section{CONCLUSIONS AND DISCUSSIONS}

This framework described represents only preliminary results. It is exploratory research that deals with new security challenges faced by medical systems in a modern distributed environment. Three issues remain to be addressed in greater depth. First, the security demands of DSMIN need to be further identified and evaluated through a detailed risk analysis review. The current method of generating these demands is empirical rather than analytical. Though the major risks have been identified, some potential risks could be hidden without an analytical model. Second, a detailed access control matrix should be generated specifically for this system according to more conventional data security guideline [16][18]. Thirdly, risk analysis and modeling [17] and subsequent validation tests based on the proposed framework should be done and evaluated by a neutral reference center before and after a prototype is developed, in order to endorse the system's security properties.

\section{ACKNOWLEDGEMENTS}

This work is funded in part by a grant from the State of Connecticut under its Critical Technology Project. Zhihong Yang wishes to extend her thanks to Dr. Howard Sholl in the University of Connecticut for partial support during this project. 


\section{REFERENCES}

1. W. Chico, Health care information systems, Communication of ACM, Vol.40, No.8, August 1997.

2. Ian R. Greenshields, Zhihong Yang, Architecture of medical imaging system, Proceedings of Eleventh IEEE symposium on computer based medical systems, pp. 132-136.

3. Zhihong Yang, A Distributed Services Medical Imaging Network, Proceedings of Twelfth IEEE symposium on computer based medical systems, pp. 18-23.

4. Betty A. Levine, Kevin Cleary and Seong K. Mun, Deployable Teleradiology: Bosnia and Beyond, IEEE Transactions on Information Technology in Biomedicine, Vol.2, No. 1, March 1998.

5. Hoehn H, Ratib O. Papyrus 3.0: the DICOM compatible file format. Geneva, Switzerland: Digital Imaging Unit, Center of Medical Informatics, University Hospital of Geneva, 1993.

6. Jene Grimson, A CORBA-Based Integration of Distributed Electronic Healthcare Records Using the Synapses Approach., etc. IEEE Transactions on Information Technology in biomedicine, Vol.2, No.3, pp124-137, Sep. 1998

7. David G. Lilman and David W. Forslund, An International Collaboratory Based on Virtual Patient Records, Communications of ACM, Vol.40, No.8, pp 110117, August 1997.

8. TeleMed, http://www.acl.lanl.gov/TeleMed/

9. T.C Ting, Privacy and Confidentiality in Healthcare Delivery Information system, Proceedings of Twelfth IEEE symposium on computer based medical systems, pp 2-5.

10. Audrey R. Chapman, Sheed and ward, Health care and information ethics: protecting fundamental human rights.

11. Thomas C. Rindfleisch, Privacy, Information Technology and Health Care, Communication of ACM, Vol.40, No.8 August 1997

12. Erik Viire, Health and Safety Issues for VR, Communication of ACM, Vol.40, No.8 August 1998.

13. Varadharajan, V.; Calvelli,C, An access control control model and its use in representing mental health application access policy, IEEE transactions on Knowledge and Data Engineering, Vol.8, No.1, pp 81-95, February 1996.

14. B.Clifford Neuman and Theodore Ts'o, Kerberos: An Authentication Service for Computer Networks, IEEE Communications Magazine, Vol.32, No.9, pages 33-38, Sep. 1994.

15. Zhihong Yang, Agent-controlled active streams in a Distributed Services Medical Imaging Network, pp 277-281, Proceedings of Twelfth IEEE symposium on computer based medical systems.

16. The SEISMED Consortium, Data Security for Healthcare, IOS Press 1996.

17. Smith E., Eloff J., "Modelling risks in a healthcare institution", in Proc. of the $15^{\text {th }}$ IFIP World Computer Congress, Vienna/Budapest, September 1998.

18. Kokolakis S., Gritzalis D., Katsikas S., "Generic Security Policies for Healthcare Information Systems", Health Informatics Journal, Vol. 4, No. 3.4, pp. 142-159, 1999. 
1.Client sees data advertised by (data) server via java-based 2: Client's request is fielded by servlet forms
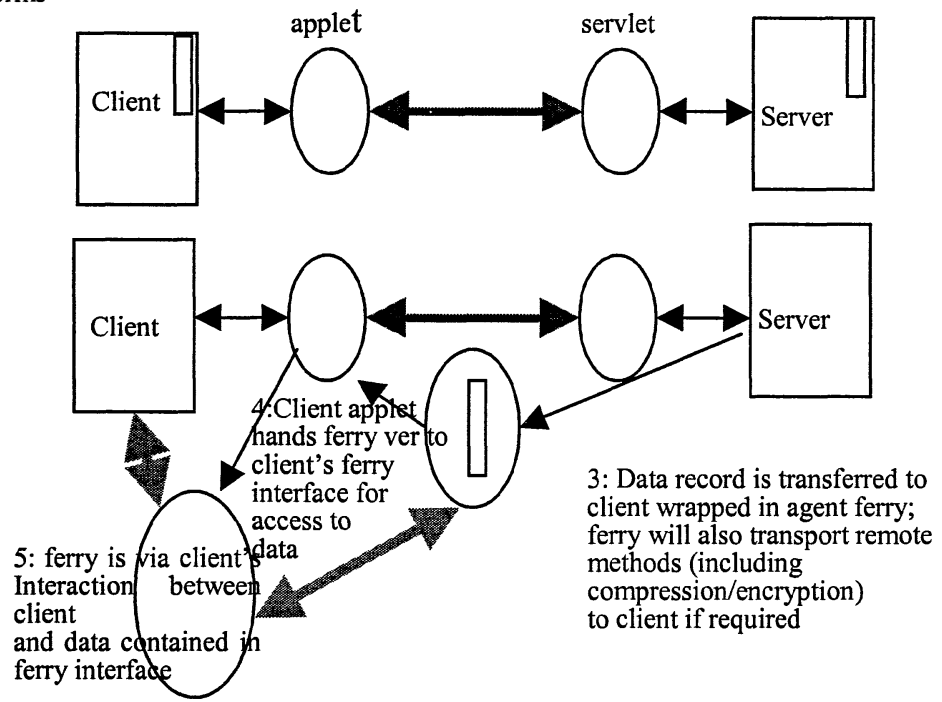

Figure 2 Transfer Protocol of Agent Ferry

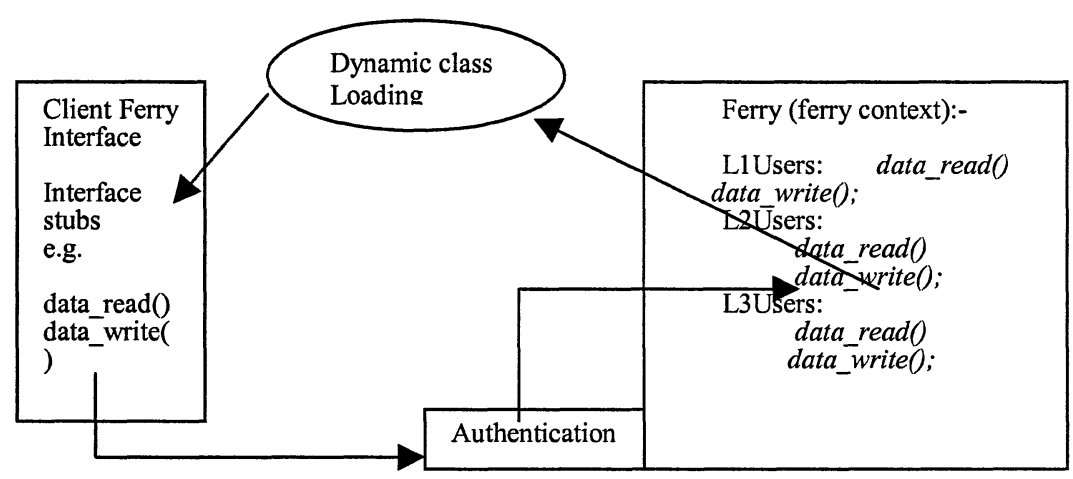

Figure 3 Name Space Interposition 